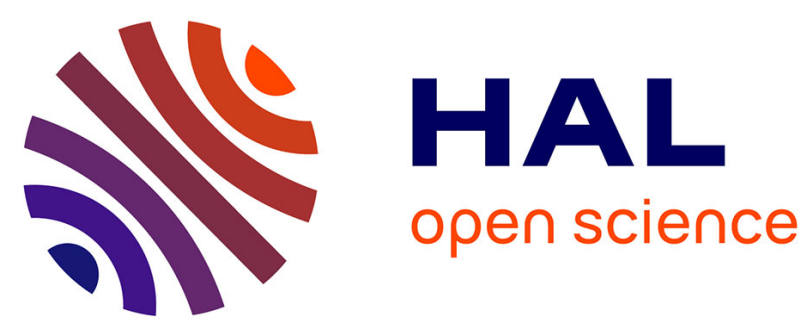

\title{
CALCUL NUMÉRIQUE DU FROTTEMENT INTÉRIEUR H: APPLICATION À MgO MONOCRISTALLIN
}

\author{
M. Gabbay, C. Esnouf, A. Vincent, Gilbert Fantozzi
}

\section{- To cite this version:}

M. Gabbay, C. Esnouf, A. Vincent, Gilbert Fantozzi. CALCUL NUMÉRIQUE DU FROTTEMENT INTÉRIEUR H: APPLICATION À MgO MONOCRISTALLIN. Journal de Physique Colloques, 1983, 44 (C9), pp.C9-569-C9-573. 10.1051/jphyscol:1983984 。 jpa-00223434

HAL Id: jpa-00223434

https://hal.science/jpa-00223434

Submitted on 1 Jan 1983

HAL is a multi-disciplinary open access archive for the deposit and dissemination of scientific research documents, whether they are published or not. The documents may come from teaching and research institutions in France or abroad, or from public or private research centers.
L'archive ouverte pluridisciplinaire HAL, est destinée au dépôt et à la diffusion de documents scientifiques de niveau recherche, publiés ou non, émanant des établissements d'enseignement et de recherche français ou étrangers, des laboratoires publics ou privés. 


\title{
CALCUL NUMÉRIQUE DU FROTTEMENT INTÉRIEUR $\triangle H$ : APPLICATION À MgO MONOCRISTALLIN
}

\author{
M. Gabbay, C. Esnouf, A. Vincent et G. Fantozzi
}

Groupe d'Etudes de Métallurgie Physique et de Physique des Matériaux, L.A.-CNRS, I.N.S.A., Bât. 502, 69621 VilZeurbanne Cedex, France

Résumé - $\Delta H$ est calculé en fonction de la température et de l'amplitude $\sigma_{\ominus}$ de la contraintede sollicitation. Pour un niveau donné $\Delta H, \sigma_{0}$ décrôit 1 inéairement avec $\mathrm{T}^{1 / 2}$ dans le domaine des basses températures. Au delà, la décroissance est toujours linéaire mais plus lente. L'extrapolation de la droite basse température définit une température $T_{C}$ caractéristique qui permet d'évaluer l'énergie de liaison entre dislocation et défaut ponctuel. Dans ce domaine basse température, le désancrage du premier défaut ponctuel suffit pour entraîner le désancrage total alors que, dans le domaine haute température, Te désancrage s'effectue collectivement à partir de plusieurs défauts ancreurs.

Abstract - $\Delta H$ is computed as a function of temperature and stress amplitude o. For a given level $\Delta H, \sigma_{0}$ decreases 7 inearly versus $\mathrm{Tl} / 2$ along the low temperature range. For higher temperature, such a linear decrease is observed but the slope is lower. The extrapolation of the low temperature straight line determine a critical temperature Tc which allows an estimation of the binding energy between dislocation and point defect. Along the low temperature range, complete breakaway is triggered by the breakaway of the first pin; at higher temperature, severals pins are collectively involved.

\section{I - INTRODUCTION}

L'étude de T'intéraction dislocations-défauts ponctuels dans Mg0 monocristallin déformé plastiquement à l'ambiante nous a conduit à mesurer le frottement intérieur dû au désancrage $\Delta H$ en fonction de l'amplitude de vibration sinusoïdale (fréquence $10 \mathrm{kHz}$ ) pour différents états de recuit. Différents modèles théoriques de désancrage ont été appliqués à ces mesures expérimentales et les premières analyses des résultats ont été publiées par ailleurs $\{1\},\{2\}$. Parmi ces modèles, il est utjle de rappeler les bases de celui issu de la théorie de LUCKE et a ${ }^{\prime \prime}\{3\}$ décrivant $\}$ e désancrage thermomécanique; dans $7 \mathrm{l}$ domaine basse température, $\Delta H$ est donné par :

(o) $\Delta H \cong 0$ pour $\sigma . \ll \sigma_{\mathrm{d} T}$

(1) $\Delta H=\Lambda L_{T}{ }^{2} \mathrm{fm}\left[\left(\sigma_{0}{ }^{2}-\sigma_{r T} T^{2}\right) / 2 \sigma_{0}{ }^{2}\right]$ pour $\sigma_{0} \leqq \sigma_{\mathrm{dT}}$

(2) $\Delta H=\Lambda L_{T} T^{2}\left[\left(\sigma_{d T} T^{2}-\sigma_{r T} T^{2}\right) / 2 \sigma_{0}^{2}\right]$ pour $\sigma_{0}>\sigma_{d T}$

avec $\Lambda$ : densité des dislocations

$\mathrm{L}_{\mathrm{T}}$ : longueur totale des segments entre ancrages durs. $\sigma_{0}$ : amplitude de 1 a contrainte $\sigma=\sigma_{0}$. sin 2 mut où $\nu$ est 1 a fréquence de sol1i-

$\sigma_{d T}, \sigma_{r T}:$ respectivement contraintes de désancrage et de réancrage thermique définies dans $\{3\}$

fm: valeur maximale de la fraction désancrée $f$ atteinte dans un demi-période $\left(\mathrm{fm}=1\right.$ lorsque $\left.\sigma_{0}>\sigma_{\mathrm{dT}}\right)$

Pour des boucles longues, $\sigma_{r T^{2}}$ devient négligeable devant $\sigma_{d T}{ }^{2}$ (car $\sigma_{\mathrm{dT}} / \sigma_{\mathrm{rT}}$ est proportionnel à $7 \mathrm{a}$ longueur des segments) et les relations (1) et (2) se simplifient. En particulier, pour la région où $\Delta H$ crồt avec $\sigma_{0}$, (1) s'écrit alors (3) $\Delta H=\Lambda L_{T}^{2} \mathrm{fm} / 2$. 
Dans cette première approche où 1 'on considère $\sigma_{r T}<<\sigma_{d T}$, le réancrage des segments désancrés peut être négligé et cette fraction maximum fóm obtenue au cours du cycle sera quasiment constante jorsque (4) Pd. $\Delta t=K$

où : - Pd est la fréquence de désancrage donnée par $P d=\nu_{d} \exp \left\{-U_{d} / k T\right\}$ (5)

$\nu_{d}$ et $U_{d}$ sont 1 a fréquence d'attaque et T'énergie d'activation du désancrage.

- $\Delta t=\alpha / \nu, \alpha$ étant une fraction de la période de mesure $1 / \nu$ pendant laquelle la contrainte agit au voisinage de son maximum $\sigma_{0}$ (LUCKE et at $\{3\}$ ).

- $K$ est une constante au maximum égale à 1 .

En utilisant la force d'intéraction linéarisêe proposée par TEUTONICO et a $1^{\prime}\{4\}$, l'énergie $U_{d}$ est donnée par :

(6) $U_{d}=U_{0}^{d}\left(1-\sigma_{d} / \sigma_{d}\right)$ avec $U_{0}$ : énergie de 1iaison dislocation-défaut ponctuel et $\sigma_{d}^{d}$ : contrainte de désancráge mécanique, on obtient une fraction désancrée constante (c'est-à-dire $\Delta \mathrm{H}$ constant) lorsque 7 a condition suivante est satis-

(7) $\sigma_{0}=\sigma_{d}\left\{1-\left(T^{1 / 2} /\left[U_{0} / k \cdot \ln \left(\alpha \cdot v_{d} / K \cdot v\right)\right] .1 / 2\right)\right\}$

Si $U_{0} / k \cdot \ln \left(\alpha \cdot v_{d} / K \cdot v\right)=C^{\text {te }}=T C$ (8) alors (7) s'écrit $\sigma_{0}=\sigma_{d}\left[1-(T / T C)^{1 / 2}\right]$

$L$ 'analyse de nos résultats expérimentaux selon le diagramme $\varepsilon\left(T^{1 / 2}\right)$ présente un bon accord avec la relation (7) vers les basses températures (Figure 1). Ainsi, nous observons lors de recuits successifs: i) une température caractéristique Tc quasiment commune montrant que l'énergia moyenne des ancreurs varie peu lors des différents traitements;

ii) le module de la pente des droites $\sigma\left(\mathrm{T}^{1 / 2}\right)$ augmente indiquant une augmentation de $\sigma_{d}, c^{\prime}$ est-à-dire une augmentation du nombre de défauts piégeant la dislocation. Au delà de ce domaine linéaire, il apparâ̂t une diminution plus lente de $\varepsilon$ avec $T 1 / 2$, mais nous $n$ 'avons pas pu déterminer si ce changement était dû à un défaut ancrant d'un autre type ou à un autre mode de désancrage. Nous avons donc entrepris un calcul numérique de $\Delta H$ afin de :

i/ préciser cette analyse et définir son domaine d'application;

ii/ interpréter le changement de pente du diagramme $\sigma(T 1 / 2)$.

\section{II - PRINCIPE DU CALCUL}

Les dislocations sont piégées par $N$ défauts ponctuels identiques répartis selon le schéma de la figure 2. Pour une telle distribution, utilisée par VINCENT et al' $\{5\}$, le défaut central définissant une double boucle symétrique est celui dont le désancrage est le plus probable. Ensuite le chemin de désancrage doit s'effectuer par désépinglages successifs des points adjacents à la grande boucle précédemment dés ancrée de longueur $L_{d}=2 L, 2 L+1,2 L+21, \ldots$ ainsi formée. Les Tongueurs totales $L_{T}$ et dés ancrées $L_{d}$, sont données par : $L_{T}=L(2+(N-1) / \beta)$ et $L_{d} \stackrel{d}{=} L\left(2+\left(N_{d}-1\right) \beta\right)$ avec $\beta=L / 1$; $N_{d}$ est le nombre de défauts adjacents désancrés. Pour $T$ et $\sigma_{0}$ donnés, $\Delta H$ est calculé à partir de 7 'aire du cycle $\varepsilon_{\text {dis }}(\sigma), \varepsilon_{\text {dis étant }}$ 1a déformation due au désancrage. Un pas de contrainte $\Delta \sigma=\sigma_{0} / \mathrm{I}$ (I: noombre de pas), choisi à priori, conduit à une variation de on escalier $\sigma(p)=(p / I) \sigma_{0}, p$ entier

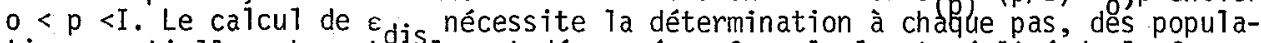
tions partiellement ou tótálement désancrées. Ce calcul est réalisé de la façon 
suivante :

i) Pour le pième pas de contrainte, le diagramme ênergétique de la dislocation est déterminé pour le chemin de désancrage le plus probable. Les énergies d'activation du désancrage $U_{d}$ (i) et du réancrage $U_{r}$ (i) du ième ancreur sont déterminées à partir du modèle de la double boucle dissymétrique de TEUTONICO et at' 44$\}$. ii) Pour le désancrage du ième point, Tes fréquences de désancrage $P_{d}(i)$ et de réancrage $P_{r}(i)$ sont alors calculées à partir de $U_{f}(i), U_{r}(i)$ et des fréquences d'attaque efficaces de désancrage et de réancrage $v_{\mathrm{d}}$ et $v_{\mathrm{r}}$ calculées par TEUTONICO et a $\}^{\prime}\{6\}$.

iii) $P_{d}(i)$ et $P_{r}(i)$ permettent le calcul

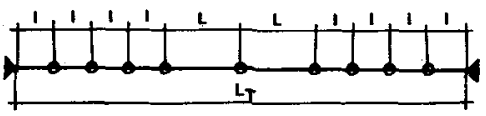

de 1a population $n_{i}+\Delta n_{j}$ des dislocations comportant $i$ points désancrés à partir des populations $n_{i}$ calculées pour le pas de contrainte précédent $(p-1)$.

(10) $\Delta n_{i}=\left[n_{(i-1)} \cdot P_{d(i-1)}+n_{(i+1)} \cdot P_{r(i+1)}-n_{i} \cdot P_{d(i)}-n_{i} \cdot P_{r(i)}\right) \Delta t$

où $\Delta t$ est la durée du pième pas de contrainte.

iii) $\Delta H$ étant obtenu, le calcul complet est réitéré pour un nombre de pas de contrainte double du précédent jusqu'à convergence satisfaisante de $\Delta H$.

$\Delta \mathrm{H}$ a ainsi été calculé pour diverses longueurs de dislocations. Ensuite, pour nous rapprocher de la réalité physique, nous avons calculé $\Delta H$ en tenant compte d'une distribution des longueurs autour d'une valeur moyenne $L \mathrm{~m}^{\circ} \Delta \mathrm{H}$ est obtenu par $1 \mathrm{a}$ relation (11) $\Delta H=\int_{L}^{\infty} \Delta H(L) . d P(L) d L$ avec $\mathcal{N}(L) d L \alpha L$ exp $\left[-\frac{L}{L_{m}}\right] d L$. L o étant la plus
courte longueur en-dessous de laquelle $\Delta H=0$.

III - RESULTATS

La figure 3 présente un réseau de courbes $\Delta H\left(\sigma_{0}\right)$ pour $L=2000 b, N=9, \beta=2$ et pour

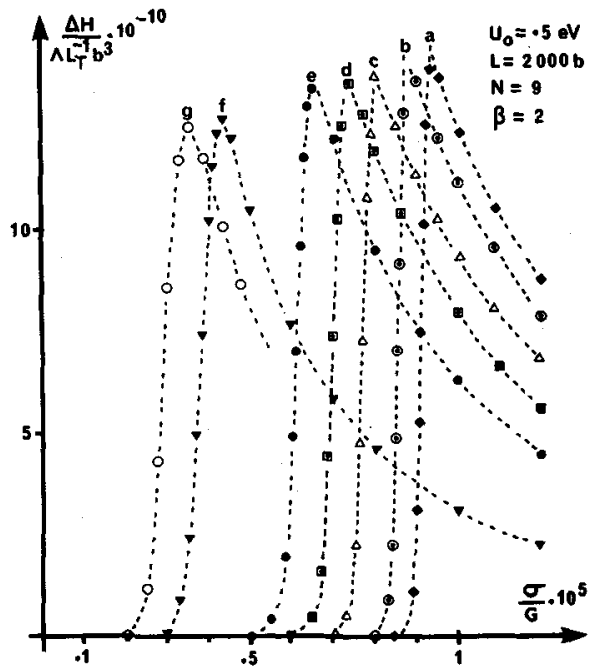

Figure $3-\Delta H$ en fonction de o pour différentes températures : $a-20 K, b-30 K, c-50 K$, d-70K, e-100K, f-200K, g-250K.

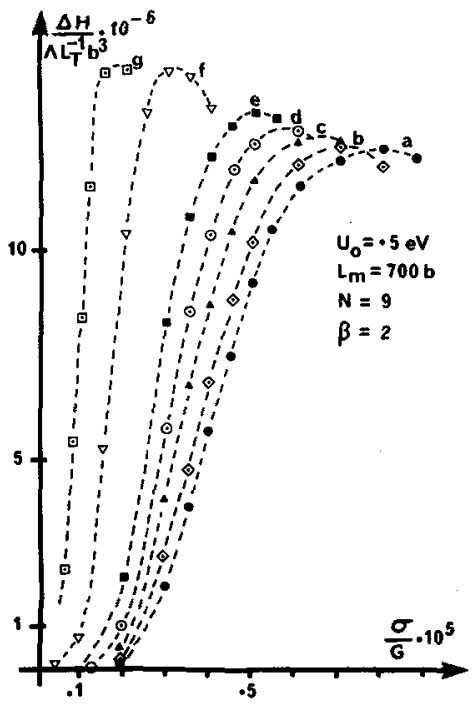

Figure $4-\Delta H$ calculé en fonction de o compte tenu d'une distribution de longueurs autour d'une longueur moyenne $L m=700 \mathrm{~b}: \mathrm{a}-20 \mathrm{~K}, \mathrm{~b}-30 \mathrm{~K}, \mathrm{c}-50 \mathrm{~K}$, d-70K, e-100K, f-200K, g-300K. 
$L=2000 b, N=9, B=2$ et pour différentes températures. Avec l'aide du tableau des populations en fonction de la contrainte, nous avons vérifié que, pour T donné, l'allure de la partie ascendante de 12 courbe $\Delta H\left(\sigma_{0}\right)$ traduit exactement l'évolution de $1 a$ fraction désancrée $f$ avec $\sigma$. Les résultats du calcul de $\Delta H$ avec distribution des longueurs sont présentés sur la figure 4. La distribution a pour effet de rendre les maximums moins aigüs et la croissance de $\Delta H$ avec $\sigma_{0}$ moins abrupte.

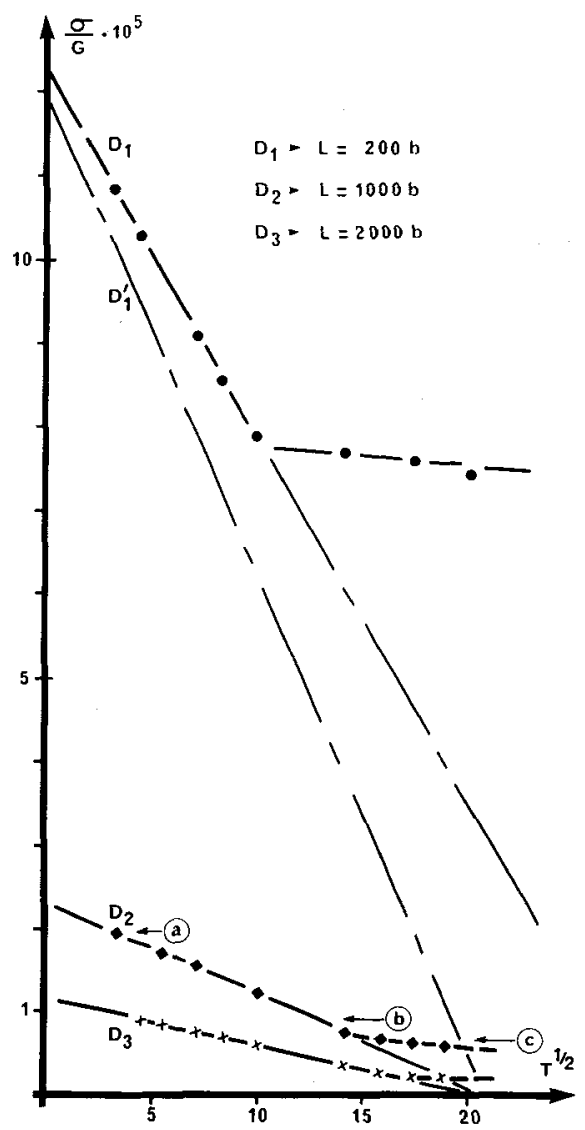

Figure 5 - Diagramme o $\left(T^{1 / 2}\right)$ pour $\Delta H$ calculé pour trois Tongueurs distinctes de dislocation et pour la même fraction dés ancrée $(f=0,5)$.

\section{IV- DISCUSSION}

La figure 5 présente les diagrammes o $(T 1 / 2)$ pour trois longueurs distinctes. Nous constatons une variation linéaire avec changement de pente sêparant basses et hautes températures : cette variation est qualitativement comparable à l'anaTyse des résultats expérimentaux. Les diagrammes énergétiques sur la figure 6 permettent d'expliquer 1'allure de 1 a variation de $\sigma$ avec $T^{1 / 2}$. Dans le domaine basse température (jusqu'au changement de pente), les diagrammes $6 a$ et $6 \mathrm{~b}$ montrent que le désancrage du premier point conditionne le désancrage de toute la ligne. Au-delà, c'est un groupe de plusieurs ancreurs qui définit la barrière énergétique à franchir pour entraîner le désancrage total (figure 6c); 1 'énergie d'activation du phénomène est alors plus élevée.

Comme l'analyse conduisant à la relation (9) Te prévoyait, nous vérifions que, du côté des basses températures, pour $\mathrm{L}=1000 \mathrm{~b}$ et $2000 \mathrm{~b}$ :

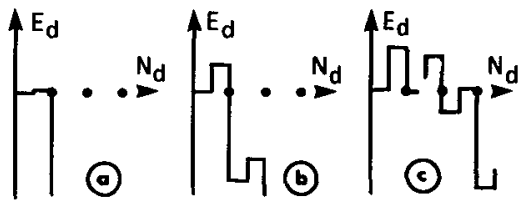

Figure 6 - Diagramme énergétique pour L=1000b et pour les contraintes $a, b, c$ repérées dans la figure 5 .

i - 1'extrapolation à $T^{1 / 2}=0$ des droites $D_{2}$ (1000b) et $D_{3}$ (2000b) conduit bien à la contrainte de désancrage mécanique du premier ancreur à savoir respectivement $\sigma_{d}=2,27.10^{-5} \mathrm{G}$ et $1,14 \cdot 10^{-5} \mathrm{G}$.

ii - la variation de 1 a pente des droites $D_{2}$ et $D_{3}$ s'effectue dans le rapport des contraintes od respectives.

ii $i$ - $T^{\prime}$ extrapolation de $D_{2}$ et $D_{3}$ à $\sigma=0$ détermine une température caractéristique

commune $T_{c}=410 \mathrm{~K}$. 
les trois observations précédentes s'expliquent par la relation (7); toutefois, précisons que, dans cette relation le coefficient $n$ 'est pas constant ma is 7 'influence de ce terme reste faible puisqu'i1 intervient en $(\ln \alpha)^{1 / 2}$.

Au sujet du cas $L=200 b$, la droite $D_{1}$ obtenue ne correspond pas à celle attendue (droite $D^{\prime} 1$ ): ce décalage et son sens ( $D^{\prime} 1 \rightarrow D_{1}$ ) s'expliquent par le fait que le modèle rappelé dans l'introduction néglige le réancrage-relation (3) - alors que cette approximation $n^{\prime e s t}$ plus valable. En effet, $\sigma_{r T}^{2} n^{\prime}$ est plus négligeable devant $\sigma_{d T}^{2}$ puisque la limite inférieure du rapport $\sigma_{r T} / \sigma_{d T} \sigma^{\top}$ est égale à $\sigma_{r} / \sigma_{d}=0,42$. Cé décalage est d'autant plus important que le rapport $\sigma_{r} / \sigma_{d T}$ est grand, c'est-àdire que la température est élevée (voir relation 1 ).

La figure 7 présente les diagrammes $\sigma\left(T^{1 / 2}\right)$ obtenus en tenant compte d'une distribution des longueurs dans le calcul de $\Delta \mathrm{H}$. Ces diagrammes, tout-à-fait semblables aux précédents, montrent que 1 a distribution $n$ 'a pas d'effet déterminant; ils ne sont en fait qu'une combinaison des droites décalées de la figure 5 . La température $T_{C}$ obtenue est de $410 \mathrm{~K}$ environ.

En vue de l'analyse des résultats expérimentaux, il est utile de préciser la relation entre $T_{C}$ et l'énergie de liaison $U_{0}$; nous avons donc déterminé, à partir des courbes $\sigma_{0}\left(T^{1 / 2}\right)$ obtenues numériquement, le coefficient $A$ te $?$ que $A=U_{0} / k T_{C}$ donc $A \simeq 14$. En utilisant cette valeur, nous déduisons pour nos résultats expérimentaux, une énergie de $1 \mathrm{i}$ aison $\mathrm{U}_{0}=0,31 \mathrm{eV}$.

$V$ - CONCLUSION

Notre calcul numérique du désancrage précise la nature des deux domaines observés dans les analyses $\sigma_{0}=f(T 1 / 2)$ à $\Delta H$ constant : le domaine basse température correspond au désancrage catastrophique à partir du premier ancreur ; le domaine haute température correspond au désancrage collectif à partir de plusieurs ancreurs. Pour le domaine loi de la forme : $\sigma_{0}=\sigma_{d}\left[1-(T / T C)^{1 / 2}\right]$.

Dans cette relation nous avons relié $T c$ a $U_{0}$ pour les boucles longues, mais $1 a$ relation reste à établir pour les boucles très courtes.

Par ailleurs, une étude plus complète est en cours afin de préciser l'effet des différents paramètres.

\section{REFERENCES}

1 - GABBAY M., FANTOZZI G., J. Phys ique C3 642 (1981) 31.

2 - GABBAY M., ESNOUF C., VINCENT A., FANTOZZI G., J. Physique C5 1042 (1981) 289.

3 - LÜCKE K., GRANATO A.V., TEUTONICO L.J., J. App. Physics 3911 (1968) 5181.

4 - TEUTONICO L.J., GRANATO A.V., LUUCKE K., J. App. Physics 351 (1964) 220.

5 - VINCENT A., PEREZ J., Phi 1. Mag. A 403 (1979) 377.

6 - GRANATO A.V., LÜCKE K., SCHLIPH J., TEUTONICO L.J., J. App. Physics 359 (1964) 2732. 